\title{
Relationship between Type 2 Diabetes Mellitus and Lumbar Bone Mineral Density in Postmenopausal Women
}

\author{
Chang-Yk Lee ${ }^{1}$, Gun-Youl Back ${ }^{1}$, Seung-Hwan Lee ${ }^{2}$ \\ ${ }^{1}$ Department of Orthopaedic Surgery, Gwangmyeong Sungae Hospital, Gwangmyeong, Korea \\ ${ }^{2}$ Department of Orthopaedic Surgery, Master Yonsei Orthopedic Clinic, Seoul, Korea
}

Study Design: Cross-sectional study using radiological measurements.

Purpose: To analyze the relationship between type 2 diabetes mellitus (DM) and bone mineral density (BMD) in postmenopausal women and to assess risk factors of osteoporotic vertebral compression fracture (OVCF) in postmenopausal diabetic women.

Overview of Literature: Type $2 \mathrm{DM}$ has negative effects on the quality of bone. Patients with type $2 \mathrm{DM}$ have increased risk of hip and other fractures, but their vertebral fracture risk is controversial. There is a positive correlation between body mass index (BMI) and BMD. At the same time, obesity is the most important risk factor for type $2 \mathrm{DM}$.

Methods: Consecutive patients whose BMD had been checked using dual-energy X-ray absorptiometry at Gwangmyung Sungae Hospital were recruited. Patients were divided into two groups according to the presence of type 2 DM. Risk factors of OVCF including age, BMI, current smoking status, current alcohol consumption, and presence of osteoporosis were analyzed separately in the type 2 DM group and control group.

Results: A total of 1,130 patients were enrolled in this study. The mean age was 63.2 years. BMI was positively correlated with lumbar BMD in the control group $(r=0.284)$ and in the diabetic group $(r=0.302)$. In subgroup analysis, $\mathrm{BMI}$ and age were significant risk factors of OVCF in the type $2 \mathrm{DM}$ group. In multiple linear regression analysis, type $2 \mathrm{DM}(\beta=0.035 ; 95 \%$ confidence interval [CI], $0.005-0.065 ; p=0.024)$ and $\mathrm{BMI}(\beta=0.015 ; 95 \% \mathrm{Cl}, 0.012-0.018 ; \beta<0.001)$ were positively correlated with lumbar $\mathrm{BMD}$, and age was negatively correlated with $\mathrm{BMD}(\beta=-0.006 ; 95 \% \mathrm{Cl},-0.007$ to $-0.004 ; p<0.001)$.

Conclusions: BMI was positively correlated with lumbar BMD and was higher in type 2 diabetic patients. Age was negatively correlated with lumbar BMD.

Keywords: Type 2 diabetes mellitus; Osteoporotic fractures; Compression fractures; Body mass index; Bone mineral density

\section{Introduction}

Diabetes and osteoporosis are major public health prob- lems that have an increased prevalence in the aging population [1,2]. Type 2 diabetes mellitus (DM) is the result of resistance to insulin and an inadequate compensatory

Received Apr 5, 2021; Revised Jun 14, 2021; Accepted Jun 17, 2021

Corresponding author: Seung-Hwan Lee

Department of Orthopaedic Surgery, Master Yonsei Orthopedic Clinic, 190, Siheung-daero, Geumcheon-gu, Seoul 08630, Korea

Tel: +82-2-807-1175, Fax: +82-2-807-1175, E-mail: java5885@gmail.com 
insulin secretion response to glucose. Besides the role of insulin in glucose metabolism, it is thought that insulin also has an anabolic effect on bone, resulting in a higher bone mineral density (BMD) [3]. Type 1 DM results from the pancreas's failure to produce enough insulin due to the loss of beta cells and has a different effect on bone.

Several studies have found that patients with type 2 diabetes are at an increased risk of hip fracture despite having higher BMD than non-diabetic individuals [4-6]. In Asians, Korean males and females with type 2 diabetes were shown to be at increased risk of hip fractures compared with non-diabetic individuals [7]. In addition, patients with diabetes have greater mortality and develop more complications than non-diabetic patients. However, the relationship between type $2 \mathrm{DM}$ and vertebral fracture risk is unclear.

There is a positive correlation between body mass index (BMI) and BMD. At the same time, obesity is the most important risk factor for type 2 diabetes. Low BMI is a well-documented risk factor for future fracture, whereas a high BMI appears to be protective. We hypothesized that lumbar BMD is higher but the prevalence of osteoporotic vertebral compression fracture (OVCF) is also higher in type $2 \mathrm{DM}$ patients. In this study, we compared the prevalence of BMD and OVCF in patients without diabetes and in patients with type $2 \mathrm{DM}$, and analyzed the correlation between $\mathrm{BMI}$ and bone density in type $2 \mathrm{DM}$ patients.

\section{Materials and Methods}

This study received an exemption by the Institutional Review Board of Gwangmyung Sungae Hospital (IRB approval no., KIRB-2021-N-005). The requirement for informed consent from individual patients was omitted because of the retrospective design of the study.

\section{Patient enrollment}

A single-center retrospective study was performed. Consecutive postmenopausal women aged $\geq 50$ years whose BMD had been checked using dual-energy X-ray absorptiometry (DEXA) at Gwangmyung Sungae Hospital were recruited between January 2014 and December 2017. The age at menopause in Korean women is 50 years and their BMD is usually checked after menopause. This is a retrospective study using previously acquired data, so we limited patients to postmenopausal women aged over 50 years.
The diabetes group included patients who had prescription records for antidiabetic medications and had been diagnosed with type $2 \mathrm{DM}$ (International Classification of Diseases, 10th revision codes E11.0-E11.9). Patients with impaired fasting glucose or impaired glucose tolerance were excluded. The severity of diabetes including complications was not assessed. However, we included type $2 \mathrm{DM}$ patients taking antidiabetic medications (oral medications and insulin) and with a duration of disease of more than 5 years. Type $2 \mathrm{DM}$ is diagnosed by demonstrating any one of the following using the World Health Organization (WHO) diabetes diagnostic criteria.

Type $1 \mathrm{DM}$ results from the pancreas's failure to produce enough insulin due to the loss of beta cells and has a different effect on bone, so we excluded patients with type $1 \mathrm{DM}$. The non-diabetic control group included patients without a type $2 \mathrm{DM}$ code diagnosis and no record of antidiabetic medication prescriptions. Exclusion criteria were as follows: (1) previous surgery on thoracolumbar spine; (2) lumbar spinal deformity resulting from fracture, tumor, infection, or congenital abnormality; (3) neuromuscular disease; (4) inflammatory arthritis including ankylosing spondylitis and rheumatoid arthritis; and (5) type $1 \mathrm{DM}$.

People in Asia tend to develop diabetes with less severe obesity and at younger ages, suffer longer with diabetic complications, and die sooner than people in other regions. These observations prompted the WHO to establish Asia-Pacific guidelines for the diagnostic criteria of obesity. The WHO concluded that a substantial proportion of Asian people with BMI lower than $25.0 \mathrm{~kg} / \mathrm{m}^{2}$ (and thus not classified as overweight under the existing WHO definition) are at high risk of type 2 diabetes and cardiovascular disease [8]. Therefore, we defined patients with BMI values above $25.0 \mathrm{~kg} / \mathrm{m}^{2}$ as being obese.

We checked risk factors of OVCF including age, BMI, current smoking status, current alcohol consumption, and presence of osteoporosis (bone mass within the last year, as determined by DEXA; using minimum values of lumbar spine [L1-L4] and femoral neck, and total hip T-score lower than -2.5) using lumbar BMD at the time of patient enrollment.

Data were obtained using a self-reported questionnaire. Those who currently smoked (current smoking here refers to those who have smoked more than 5 packs [n=100] of cigarettes in their lifetime and now smoke regularly; refer to the National Health Statistics Standards) were regarded 
as smokers [9]. Alcohol consumption was measured using the AUDIT (Alcohol Use Disorders Identification Test) scoring system [10]. Those consuming more than 1 unit per week of alcohol were regarded as current alcohol consumers. BMI (weight $[\mathrm{kg}] /$ height $[\mathrm{m}]^{2}$ ) was measured using an automatic height, weight, and obesity meter by Xenix (DS-1003), and the average value was measured twice per subject by an orthopedic nurse.

\section{Radiological assessment}

Standing antero-posterior, lateral, flexion, and extension radiographs (1/2P13DK-85; Shimadzu, Kyoto, Japan) of the lumbar spine were performed. A lateral radiograph of the lumbar spine was obtained in a comfortable standing position with the upper extremities placed naturally at the sides of the trunk and the head facing forward to maintain a horizontal gaze. Measurements were performed using computer software (Infinitt PiViewSTAR 5051 workstation; Infinitt Healthcare Co. Ltd., Seoul, Korea), and the contrast adjustment feature of the digital system was used. Vertebral fractures were defined using Genant's semiquantitative method (combination of morphometric and visual assessments), which is commonly used for diagnosing vertebral fractures [11].

Patients whose BMD had been checked using DEXA (Prodigy Bone Densitometry; GE Medical Systems Lunar, Madison, WI, USA) at our institute were recruited between January 2014 and December 2017 and the diagnosis of vertebral fracture was performed in this period. DEXA BMD $\left(\mathrm{g} / \mathrm{cm}^{2}\right)$ was measured using fan-beam DEXA (QDR 4500W; Hologic Inc., Waltham, MA, USA) and using standardized procedures and centralized quality control procedures. Patients with acute vertebral fracture diagnosed using magnetic resonance imaging (MRI) were enrolled during this period and the diagnosis of type 2 DM proceeded the diagnosis of vertebral fracture.

Analyses of radiographic data were performed by two experienced orthopedic surgeons. After 1 week, repeated, blinded evaluation of MRI images and radiographs from 100 patients was performed by two orthopedic surgeons to obtain measures for calculating the intra- and interrater reliability. In terms of the intra- and inter-rater reliability between the readers, a high degree of consensus (range, 0.79-0.95) was previously identified for another cohort using MRI images and radiographs.

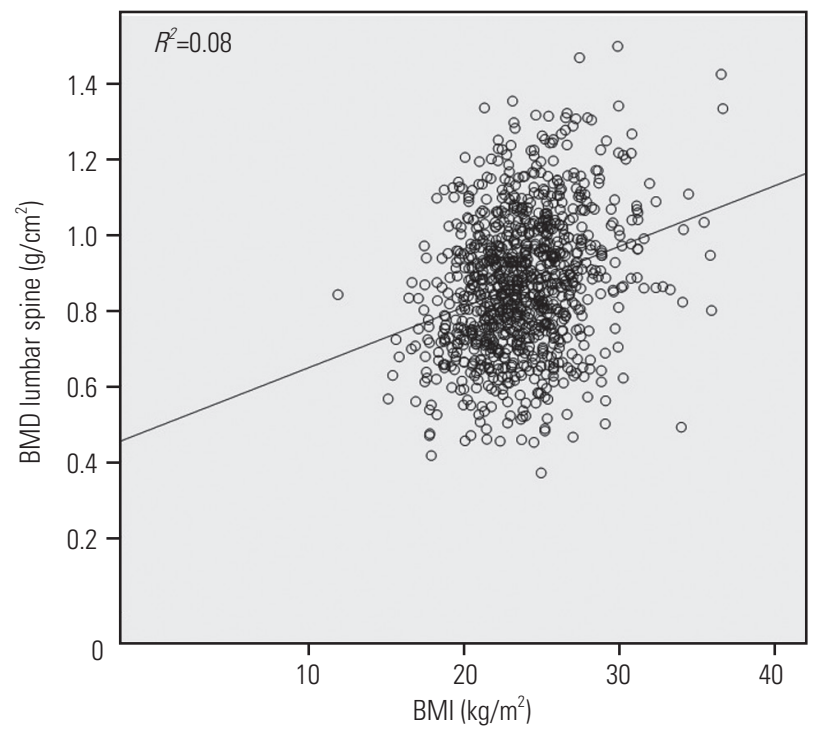

Fig. 1. Correlation analysis between bone mineral density (BMD) and body mass index (BMI) in control group (Pearson $r=0.284$ ).

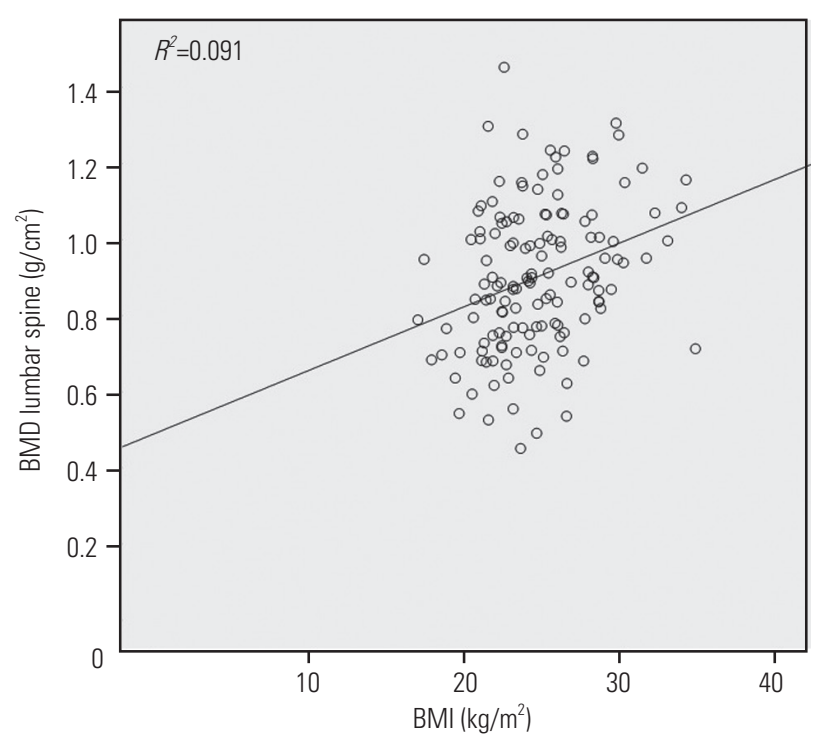

Fig. 2. Correlation analysis between bone mineral density (BMD) and body mass index (BMI) in diabetes mellitus group (Pearson $r=0.302$ ).

\section{Statistical analysis}

Patients were divided into two groups according to the presence of type $2 \mathrm{DM}$. BMD and the prevalence of OVCF were compared between the type $2 \mathrm{DM}$ group and the control group. The risk factors of OVCF including age, BMI, current smoking status, current alcohol consumption, and presence of osteoporosis as determined using lumbar BMD were analyzed separately in the type $2 \mathrm{DM}$ group and the control group. Correlations between BMI 
and BMD were analyzed using Pearson's correlation test (Figs. 1, 2).

We confirmed the normal distribution of all continuous variables using the Kolmogorov-Smirnov method. Multivariable logistic regression analysis was performed to determine the adjusted odds ratios (ORs) of fractures after adjusting for multiple confounders. All statistical analyses were performed using the SPSS ver. 17.0 statistics package (SPSS Inc., Chicago, IL, USA). A value of $p<0.05$ was accepted as significant.

\section{Results}

A total of 1,130 patients were enrolled in this study. The mean age was 63.2 years (range, 60-90 years). Overall, 139 patients had type $2 \mathrm{DM}$. The average BMI of the patients was $23.87 \mathrm{~kg} / \mathrm{m}^{2}$. The lumbar BMD was $0.87 \mathrm{~g} / \mathrm{cm}^{2}$. The prevalence of OVCF was $40.3 \%$ and the prevalence of osteoporosis was $16.6 \%$ (Table 1).

\section{Correlation analysis between bone mineral density and body mass index}

BMI was positively correlated with lumbar BMD in both the diabetic group $(r=0.302, p<0.001)$ and the control group $(r=0.284, p<0.001)$ (Figs. 1, 2).

\section{Subgroup analysis of control group}

Multivariable logistic regression analysis revealed that age (OR, 1.07; 95\% confidence interval [CI], 1.05-1.09; $p<0.001)$ and osteoporosis (OR, 2.23; 95\% CI, 1.54-3.21; $p<0.001)$ were significant risk factors of OVCF in the con-

Table 1. Descriptive statistics of all patients $(\mathrm{N}=1,130)$

\begin{tabular}{lc} 
Characteristic & Value \\
\hline Age $(\mathrm{yr})$ & $63.22 \pm 7.85$ \\
\hline Body mass index $\left(\mathrm{kg} / \mathrm{m}^{2}\right)$ & $23.87 \pm 3.25$ \\
\hline L-BMD $\left(\mathrm{g} / \mathrm{cm}^{2}\right)$ & $0.87 \pm 0.20$ \\
\hline L-BMD (T-score) & $-1.85 \pm 1.42$ \\
\hline Osteoporotic vertebral compression fracture & $456(40.3)$ \\
\hline Osteoporosis & $188(16.6)$ \\
\hline Current alcohol consumer & $269(23.8)$ \\
\hline Current smoker & $62(5.5)$ \\
\hline
\end{tabular}

Values are presented as mean \pm standard deviation or number (\%). L-BMD, lumbar bone mineral density. trol group after adjusting for BMI, alcohol consumption status, and current smoking status (Table 2).

\section{Subgroup analysis of diabetic group (type 2 diabetes mellitus)}

Multivariable logistic regression analysis revealed that BMI (OR, 1.14; 95\% CI, 1.02-1.26; $p=0.017)$ and age (OR, 1.05; 95\% CI, 1.00-1.11; $p=0.033$ ) were significant risk factors of OVCF in the type $2 \mathrm{DM}$ group after adjusting for alcohol consumption status, current smoking status, and osteoporosis (Table 3). It was not clarified that osteoporosis was a significant risk factor for OVCF $(p=0.469)$, which contrasted with the findings in the control group.

\section{Relationship between type 2 diabetes mellitus and osteoporosis}

We performed logistic regression analysis to analyze the relationship between type $2 \mathrm{DM}$ and osteoporosis according to lumbar BMD (Table 4). Type 2 DM was not shown to be a risk factor for osteoporosis. However, increased BMI (OR, 0.90; 95\% CI, $0.86-0.95$; $p<0.001$ ) was shown to lower the risk of osteoporosis. In addition, increased age (OR, 1.09; $95 \%$ CI, $1.07-1.17$; $p<0.001)$ was a significant risk factor for osteoporosis after adjusting for type $2 \mathrm{DM}$ status, alcohol consumption status, and current smoking status.

Table 2. Subgroup analysis using logistic regression (control group)

\begin{tabular}{lcr} 
Variable & Odds ratio (95\% confidence interval) & $p$-value \\
\hline Body mass index & $0.98(0.94-1.03)$ & 0.426 \\
\hline Current alcohol consumer & $1.03(0.75-1.42)$ & 0.835 \\
\hline Current smoker & $1.24(0.69-2.24)$ & 0.469 \\
\hline Age & $1.07(1.05-1.09)$ & $<0.001$ \\
\hline Osteoporosis & $2.23(1.54-3.21)$ & $<0.001$ \\
\hline
\end{tabular}

Table 3. Subgroup analysis using logistic regression (diabetic group: type 2 diabetes mellitus)

\begin{tabular}{lcc} 
Variable & Odds ratio $(95 \%$ confidence interval) & $p$-value \\
\hline Body mass index & $1.14(1.02-1.26)$ & 0.017 \\
Current alcohol consumer & $0.669(0.27-1.64)$ & 0.380 \\
Current smoker & $1.381(0.30-6.38)$ & 0.679 \\
Age & $1.05(1.00-1.11)$ & 0.033 \\
Osteoporosis & $0.70(0.55-3.73)$ & 0.469
\end{tabular}


In multiple linear regression analysis, BMI was positively correlated with lumbar BMD, and was higher in type 2 diabetic patients. Meanwhile, age was negatively correlated with lumbar BMD (Table 5).

\section{Discussion}

This study showed that lumbar spine BMD in type 2 diabetic patients was higher than that in control patients. Age and increased BMI are important risk factors of OVCF in type 2 diabetic patients.

Diabetes is one of the fasting growing diseases in the western world and is also becoming a major problem in countries with emerging economies such as India and China. The numbers of patients are staggering, and the rate of emergence of new diabetics continues to grow, especially as type $2 \mathrm{DM}$ is correlated with obesity and metabolic syndrome, which are themselves becoming global health problems [12].

Diabetes is associated with an increased risk of fracture, although type $2 \mathrm{DM}$ is often characterized by normal or high BMD. Thus, diabetes may be correlated with a reduction of bone strength, which is not reflected in the measurement of BMD. This phenomenon in type 2 diabetes may be explained by poorer "bone quality" rather than

Table 4. Analysis between type $2 \mathrm{DM}$ and osteoporosis using logistic regression

\begin{tabular}{lcr} 
Variable & Odds ratio (95\% confidence interval) & $p$-value \\
\hline Type 2 DM & $0.95(0.58-1.55)$ & 0.825 \\
Body mass index & $0.90(0.86-0.95)$ & $<0.001$ \\
Current alcohol consumer & $0.72(0.48-1.09)$ & 0.117 \\
Current smoker & $1.87(1.01-3.48)$ & 0.048 \\
Age & $1.09(1.07-1.12)$ & $<0.001$ \\
\hline
\end{tabular}

DM, diabetes mellitus.

Table 5. Linear regression analysis between lumbar bone mineral density and risk factors

\begin{tabular}{lcr} 
Variable & $\beta(95 \%$ confidence index $)$ & $p$-value \\
\hline Type 2 diabetes mellitus & $0.035(0.005$ to 0.065$)$ & 0.024 \\
\hline Body mass index & $0.015(0.012$ to 0.018$)$ & $<0.001$ \\
\hline Current alcohol consumer & $-0.022(-0.045$ to 0.002$)$ & 0.067 \\
Current smoker & $-0.022(-0.065$ to 0.022$)$ & 0.326 \\
\hline Age & $-0.006(-0.007$ to -0.005$)$ & $<0.001$ \\
\hline
\end{tabular}

bone mass.

Bone is a complex tissue whose principal function is to resist mechanical forces and fracture. Bone strength depends not only on the quantity of bone tissue but also on the quality, which is characterized by the geometry and shape of bones, the microarchitecture of the trabecular bones, turnover, minerals, and collagen [13].

In this study, lumbar BMD was significantly higher in the diabetic group. In subgroup analysis of the diabetic group, there was no significant difference of lumbar BMD between the fracture group and the control group. In the patients with diabetes, obesity (BMI $\left.\geq 25.0 \mathrm{~kg} / \mathrm{m}^{2}\right)$ was a significant risk factor for OVCF. This can be explained by the poor bone quality of diabetic patients who were overweight. The poor bone quality of diabetic patients is due to metabolic changes in collagen formation and mineralization of the bone cortex.

Animal and clinical studies have provided evidence that the accumulation of advanced glycation end products (AGEs) in diabetic bone collagen contributes to reduced material properties and greater susceptibility to fracture. Higher levels of circulating AGEs are reported to increase the risk of fracture [14]. The accumulation of AGEs leads to defective collagen via the formation of irreversible cross-links between the fibers in the triple helix [15].

Collagen cross-linking plays an important role in bone strength $[13,16]$. It involves the enzymatic action of lysyl oxidase $(p=0.469)$, which leads to the formation of immature and mature cross-links that stabilize the collagen fibrils. In diabetic patients, two type of non-enzymatic process in type I collagen have been described: the formation of advanced glycation end products due to the accumulation of reducible sugars in bone tissue, and the process of racemization and isomerization in collagen telopeptides. These collagen modifications impair the mechanical properties of bone in diabetic patients [13].

With regard to cortical bone, Patsch et al. [17] found increased cortical porosity in diabetic postmenopausal women with prevalent fracture, compared with that in diabetic women without a fracture history, using highresolution peripheral quantitative computed tomography. The cortex of the distal radius in type 2 diabetic patients with distal radius fracture had 4.7 -fold greater relative porosity than that in DM patients without fracture. Severe deficits in cortical bone quality have been reported to be responsible for fragility fractures in postmenopausal diabetic women [17]. High porosity in the mid-cortical 
and periosteal layers in the high-risk type 2 DM group suggested that these cortical zones might be particularly susceptible to type $2 \mathrm{DM}$-induced toxicity and may reflect cortical micro-angiopathy [18]. Therefore, in overweight diabetic patients, bone is more fragile than would be expected for a given bone density by DEXA.

Serum P1NP (procollagen type I intact N-terminal propeptide) and serum CTX (C-terminal cross-linking telopeptide of type I collagen) as markers of bone formation and resorption have been reported in type $2 \mathrm{DM}$ [19]. Patients with type $2 \mathrm{DM}$ have lower bone turnover than non-DM controls. The underlying defect in type $2 \mathrm{DM}$ appears to be predominantly a quality-related problem coupled with a low turnover state by the suppression of markers of bone formation and resorption. These changes of bone metabolism in type $2 \mathrm{DM}$ are referred to as diabetic osteodystrophy.

In this study, obesity (BMI $\geq 25.0 \mathrm{~kg} / \mathrm{m}^{2}$ ) was a significant risk factor for vertebral fracture in subgroup analysis of the diabetic group. Most of the available evidence supports a lower risk of proximal femur and vertebral fracture in obese adults [20]. A positive correlation between BMI and BMD has been reported [21]. One possible mechanism for the higher BMD in obesity is increased mechanical loading and strain. In addition, circulating leptin acts on bone cells directly to increase bone formation [22].

However, obesity is not entirely protective against fracture and has some site-specific effects on it. An increase in BMI is associated with a decrease in the risk of vertebral fracture in men, but not in women, suggesting possible sex differences in this relationship [23]. It is possible that, even if BMD increases in response to obesity, the capacity for such an increase is limited and eventually the load-tostrength ratio (i.e., the ratio of the applied impact force to the bone strength; when its value crosses a certain threshold, fracture occurs) rises enough to cause fracture upon low-trauma injuries. In diabetic patients in our study, the quality of bone was impaired and the increase of the loadto-strength ratio with overweight may have resulted in more vertebral fractures. Therefore, obesity is not a factor protective against vertebral fracture, but rather a risk factor.

Patients with DM had more pain, co-morbidities, and poorer health status preoperatively than those without diabetes [24]. Cardiac and renal failures were more common in patients with diabetes among those with hip fractures. The prevention of fracture is important for the quality of life and for lowering co-morbidities in type 2
DM patients. Reduction in weight and the maintenance of a normal BMI are important for preventing vertebral fractures in type $2 \mathrm{DM}$ patients.

In this study, the subgroup analysis of diabetic patients showed that the proportion undergoing treatment for osteoporosis was significantly higher in the fracture group. Most antiresorptives suppress the frequency of activation of cortical bone and decrease bone turnover, thus posing a problem in the management of diabetic osteodystrophy. It appears logical that, in a disease with low bone turnover, anabolic therapy should be the treatment of choice, but data on its use in such a patient population are currently lacking. In addition, treatment guidelines for diabetic osteodystrophy should be established based on future data.

There are some limitations in our study. First, our study is cross-sectional in nature and the prevalence of OVCF was evaluated. Prospective study will be needed to evaluate the exact incidence of OVCF in type 2 diabetic patients. Second, the severity and duration of type 2 diabetes were not considered when analyzing the relationship between type $2 \mathrm{DM}$ and BMD. Third, patient enrollment was restricted to postmenopausal women, so we could not analyze the effects of type 2 diabetes on OVCF in the general population. Lastly, this study was performed retrospectively in a single center in a cross-sectional format and the study population was confined to postmenopausal women.

\section{Conclusions}

BMI was positively correlated with lumbar BMD, and it was higher in type 2 diabetic patients. Age was negatively correlated with lumbar BMD. The clinical implication of our study is that the correlation between type $2 \mathrm{DM}$ and lumbar BMD was statistically significant and risk factor of OVCF was evaluated. Type 2 DM patients, even those with normal BMD, should be aware of the risk of OVCF. The risk factor of OVCF in type $2 \mathrm{DM}$ patients can be evaluated via a prospective study.

\section{Conflict of Interest}

No potential conflict of interest relevant to this article was reported.

\section{References}

1. Cauley JA. Public health impact of osteoporosis. J 
Gerontol A Biol Sci Med Sci 2013;68:1243-51.

2. Jang HD, Kim EH, Lee JC, Choi SW, Kim K, Shin BJ. Current concepts in the management of osteoporotic vertebral fractures: a narrative review. Asian Spine J 2020;14:898-909.

3. Yano H, Ohya K, Amagasa T. Effects of insulin on in vitro bone formation in fetal rat parietal bone. Endocr J 1994;41:293-300.

4. De Liefde II, van der Klift M, de Laet CE, van Daele PL, Hofman A, Pols HA. Bone mineral density and fracture risk in type-2 diabetes mellitus: the Rotterdam Study. Osteoporos Int 2005;16:1713-20.

5. Martinez-Laguna D, Tebe C, Javaid MK, et al. Incident type 2 diabetes and hip fracture risk: a population-based matched cohort study. Osteoporos Int 2015;26:827-33.

6. Melton LJ 3rd, Leibson CL, Achenbach SJ, Therneau TM, Khosla S. Fracture risk in type 2 diabetes: update of a population-based study. J Bone Miner Res 2008;23:1334-42.

7. Kim SH, Kim YM, Yoo JS, Choe EY, Kim TH, Won YJ. Increased risk of hip fractures in Korean patients with type 2 diabetes: a 6-year nationwide populationbased study. J Bone Miner Metab 2017;35:623-9.

8. Yoon KH, Lee JH, Kim JW, Cho JH, Choi YH, Ko SH, et al. Epidemic obesity and type 2 diabetes in Asia. Lancet 2006;368:1681-8.

9. World Health Organization. Monitoring tobacco use. In: World Health Organization, editor. Guidelines for controlling and monitoring the tobacco epidemic. Geneva: World Health Organization; 1998. p. 76-8.

10. Saunders JB, Aasland OG, Babor TF, de la Fuente JR, Grant M. Development of the Alcohol Use Disorders Identification Test (AUDIT): WHO collaborative project on early detection of persons with harmful alcohol consumption--II. Addiction 1993;88:791-804.

11. Genant HK, Wu CY, van Kuijk C, Nevitt MC. Vertebral fracture assessment using a semiquantitative technique. J Bone Miner Res 1993;8:1137-48.

12. Epstein S, Defeudis G, Manfrini S, Napoli N, Pozzilli P; Scientific Committee of the First International Symposium on Diabetes and Bone. Diabetes and disordered bone metabolism (diabetic osteodystrophy): time for recognition. Osteoporos Int 2016;27:1931-51.

13. Viguet-Carrin S, Garnero P, Delmas PD. The role of collagen in bone strength. Osteoporos Int 2006;17:31936.
14. Schwartz AV, Sellmeyer DE. Diabetes, fracture, and bone fragility. Curr Osteoporos Rep 2007;5:105-11.

15. Viguet-Carrin S, Roux JP, Arlot ME, et al. Contribution of the advanced glycation end product pentosidine and of maturation of type I collagen to compressive biomechanical properties of human lumbar vertebrae. Bone 2006;39:1073-9.

16. Saito M, Fujii K, Soshi S, Tanaka T. Reductions in degree of mineralization and enzymatic collagen crosslinks and increases in glycation-induced pentosidine in the femoral neck cortex in cases of femoral neck fracture. Osteoporos Int 2006;17:986-95.

17. Patsch JM, Burghardt AJ, Yap SP, et al. Increased cortical porosity in type 2 diabetic postmenopausal women with fragility fractures. J Bone Miner Res 2013;28:313-24.

18. Heilmeier U, Cheng K, Pasco C, et al. Cortical bone laminar analysis reveals increased midcortical and periosteal porosity in type 2 diabetic postmenopausal women with history of fragility fractures compared to fracture-free diabetics. Osteoporos Int 2016;27:2791802.

19. Purnamasari D, Puspitasari MD, Setiyohadi B, Nugroho $\mathrm{P}$, Isbagio $\mathrm{H}$. Low bone turnover in premenopausal women with type 2 diabetes mellitus as an early process of diabetes-associated bone alterations: a crosssectional study. BMC Endocr Disord 2017;17:72.

20. De Laet C, Kanis JA, Oden A, et al. Body mass index as a predictor of fracture risk: a meta-analysis. Osteoporos Int 2005;16:1330-8.

21. Yang S, Shen X. Association and relative importance of multiple obesity measures with bone mineral density: the National Health and Nutrition Examination Survey 2005-2006. Arch Osteoporos 2015;10:14.

22. Cornish J, Callon KE, Bava U, et al. Leptin directly regulates bone cell function in vitro and reduces bone fragility in vivo. J Endocrinol 2002;175:405-15.

23. Kaze AD, Rosen HN, Paik JM. A meta-analysis of the association between body mass index and risk of vertebral fracture. Osteoporos Int 2018;29:31-9.

24. Ekstrom W, Al-Ani AN, Saaf M, Cederholm T, Ponzer S, Hedstrom M. Health related quality of life, reoperation rate and function in patients with diabetes mellitus and hip fracture: a 2 year follow-up study. Injury 2013;44:769-75. 